\title{
Composición y diversidad de especies forestales en el páramo Machay- Chimborazo- Ecuador
}

Composition and diversity of forest species in Machay paramo - ChimborazoEcuador

Mayra Janeth Paguay Tingo ${ }^{1}$, Daniel Arturo Román Robalino ${ }^{2}$ \& Jorge Patricio Cevallos Rodríguez ${ }^{3}$

Recibido: 19-04-2019 / Revisado: 25-05-2019 /Aceptado: 28-06-2019/ Publicado: 15-07-2019

\begin{abstract}
.
DOI: https://doi.org/10.33262/cienciadigital.v3i3.1.706

The present work aims to inventory the floristic diversity of the Machay paramo ecosystem, located in the Ilapo parish, Guano Canton, Chimborazo Province, field trips were made to georeference the territory and install five transitory plots of $25 \mathrm{~m} 2$ with 4 subplots of $1 \mathrm{~m} 2$, from which the data record was obtained for the identification, quantification and analysis of floristic diversity. In the inventory were recorded: 40 samples of terrestrial vascular plants, corresponding to 16 botanical families, 36 genera and 40 species, in addition a sample of lichen and moss of unknown family, genus and species was collected, which adds 42 plant samples. The inventory allowed to determine that the moss is the most representative species in all the plots and quadrants. According to the Shannon index, plot five possesses high diversity that is corroborated with the results obtained from the Simpson index, results that showed high values in relation to the other plots. The results of Sorensen show that the plots (P3 vs P5 and P1 vs P5) are very similar to each other. Concluding that this type of research contributes to the making of strategic decisions that allow the conservation, protection, and management of the MACHAY paramo ecosystem through the floristic inventory.
\end{abstract}

\footnotetext{
${ }^{1}$ Escuela Superior Politécnica de Chimborazo, Carrera de Ingeniería Forestal, Riobamba, Ecuador. Tesista mayra201223@hotmail.com

${ }^{2}$ Escuela Superior Politécnica de Chimborazo, Facultad de Recursos Naturales. Riobamba, Ecuador. daniel.roman@espoch.edu.ec

3 Escuela Superior Politécnica de Chimborazo, Facultad de Recursos Naturales. Riobamba, Ecuador. jorge.cevallos@espoch.edu.ec
} 
Keywords: Indices, Inventory, Shannon-Weaver, Simpson, Sorensen.

\section{Resumen.}

El presente trabajo tiene por objetivo inventariar la diversidad florística que posee el ecosistema páramo Machay, ubicado en la parroquia Ilapo, Cantón Guano, Provincia de Chimborazo, se realizaron salidas de campo para georreferenciar el territorio e instalar cinco parcelas transitorias de $25 \mathrm{~m} 2$ con 4 subparcelas de $1 \mathrm{~m} 2$, de las que se obtuvo el registró de datos para la identificación, cuantificación y análisis de la diversidad florística. En el inventario se registraron: 40 muestras de plantas vasculares terrestres, correspondientes a 16 familias botánicas, 36 géneros y 40 especies, además se colectó una muestra de liquen y musgo de familia, género y especie no identificados lo que suman 42 muestras vegetales. El inventario permitió determinar que el musgo es la especie más representativa en todas las parcelas y cuadrantes. De acuerdo al índice de Shannon, la parcela cinco posee diversidad alta que se corrobora con los resultados obtenidos del índice de Simpson, resultados que arrojaron valores altos en relación a las demás parcelas. Los resultados de Sorensen demuestran que las parcelas (P3 vs P5 y P1 vs P5) son muy similares entre sí. Concluyendo que este tipo de investigaciones contribuyen a la toma de decisiones estratégicas que permitan la conservación, protección, y manejo del ecosistema páramo MACHAY a través del inventario florístico.

Palabras claves: Índices, Inventario, Shannon-Weaver, Simpson, Sorensen.

\section{Introducción.}

Los páramos forman parte de una notable biodiversidad a escala de ecosistemas que se presentan en el Ecuador gracias a tres factores principales: la situación ecuatorial, la presencia de la cordillera de los Andes y otras sierras menores, la existencia de una fuente húmeda amazónica y de varias corrientes frías y cálidas frente a las costas (Medina \& Mena, 2001).

Los páramos ecuatorianos se han caracterizado por una elevada biodiversidad y un alto endemismo lo cual ayuda a la formación de más de un ecosistema (Baquero, Sierra, Ordóñez, \& Tipán, 2004).

Las mayores presiones sobre los páramos fueron a partir de los años de 1960, el agotamiento de las tierras y el fracaso de la redistribución de tierras con la reforma agraria en los años 1964 y 1973 que conllevo a ejercer una creciente presión demográfica especialmente de la población campesina sobre estos ecosistemas altos andinos. 
Es por esto que el páramo es un ecosistema, donde se desarrollan un sinnúmero de relaciones entre seres vivos y un medio ambiente con características especiales como: temperatura, humedad, radiación solar, presión atmosférica, otros (Bernal, Sánchez, \& Zapatta, 2000).

Hoy en día el ecosistema páramo no es valorado adecuadamente, en el caso particular del páramo Machay perteneciente a la regional Ilapo-Guano, se conoce que el territorio aporta principalmente agua para el consumo del ser humano a través de los sistemas de agua entubada que beneficia a varias comunidades del Cantón Guano. La Regional Ilapo-Guano en su afán de unir esfuerzos para la conservación del agua, se han aliado con juntas administradoras de agua potable de otras jurisdicciones parroquiales y han conformado la Pre-Corporación de Regionales en Defensa del Igualata que agrupa 20 comunidades, estas comunidades han reconocido la importancia de este ecosistema como regulador hídrico. En este espacio cabe mencionar que al momento de iniciar la investigación no se contaba con un monitoreo de la flora de este páramo, mucho menos con el valor de importancia florística que este territorio presenta (Plan de Desarrollo y Ordenamiento Territorial de la Parroquia de ILAPO (PD Y OT), 2016).

Debido a la importancia de este ecosistema y los pocos datos existentes, se ejecuta el presente trabajo de investigación con el fin de generar información sobre la diversidad de este lugar, y a partir de los resultados valorar la riqueza florística del sitio, para que sea un instrumento cuya información permita la toma de decisiones y se pueda actualizar el Plan de Manejo existente.

\section{Metodologia.}

\section{Caracterización del lugar}

El Páramo Machay se encuentra ubicado en la Parroquia Ilapo - Cantón Guano - Provincia de Chimborazo este páramo se considera como una zona de recarga hídrica pues forma parte de la Regional Ilapo - Guano, además este sitio de estudio pertenece a la demarcación hidrográfica del Pastaza, sub cuenca del río Chambo.

Tabla 1. Ubicación geográfica

\begin{tabular}{|l|l|l|}
\hline LUGAR DE ESTUDIO & LONGITUD & LATITUD \\
\hline REGIONAL & 769488 & 9817752 \\
\hline
\end{tabular}

Fuente: Investigación primaria, 2018

Características climáticas. - La zona se caracteriza por tener la presencia de neblina, con poca precipitación. Temperatura media: $11^{\circ} \mathrm{C}$, máxima: $25^{\circ} \mathrm{C}$ y mínima: $1^{\circ} \mathrm{C}$

Pendientes de $10 \%$ hasta $75 \%$ (Plan de Manejo de los Recursos Naturales de la Regional Ilapo-Guano 2014, pg.11). 
El rango de precipitación en todo el páramo, está entre 700 y $3.000 \mathrm{~mm}$ por año. La humedad relativa tiene un rango entre 25 y $100 \%$, con un promedio de 70-85\% (Alvarado \& Gavilanes, 2012).

Clasificación ecológica. - Según el Sistema de Clasificación de los Ecosistemas del Ecuador, realizado por el Ministerio del Ambiente en el año 2012 podemos distinguir 3 zonas de vida: Bosque siempre verde montano: 2000 - $3000 \mathrm{msnm}$

Bosque siempre verde montano alto: 3000 - $3700 \mathrm{msnm}$

Montano alto superior de páramo: 3700 - 4200 msnm., (Ministerio del Ambiente del Ecuador.[MAE], 2013).

Zonificación. - La georreferenciación se realizó en compañía de dirigentes de la PreCorporación, las coordenadas fueron tomadas con un GPS garmín. Las coordenadas registradas se ingresaron en el programa Arcgis 10.1 en el cual también se ingresó la Ortofoto Guano. Con todos los datos ingresados se dibujó el polígono de la zona de estudio y se ubicó las coordenadas de cada parcela.

\section{Instalación de Parcelas}

Para la instalación de parcelas se tomó en cuenta la metodología propuesta por (Pauli, y otros, 2015) para el Proyecto Gloria tomando en cuenta algunas modificaciones para adaptar a los páramos andinos.

Fueron instaladas 5 parcelas transitorias de $5 \mathrm{mx} 5 \mathrm{~m}\left(25 \mathrm{~m}^{2}\right)$, con una distancia de $300 \mathrm{~m}$ cada de ellas. Cada cuadrante se dividió en sub-cuadrantes de $1 \mathrm{~m}$ X 1m, las observaciones de vegetación se llevaron a cabo únicamente en los cuadrantes de las esquinas ya que los otros se alteran con el pisoteo, en la recolección de las muestras y datos. Se obtuvo datos de vegetación de los 20 cuadrantes de $1 \mathrm{~m}$ X 1m.

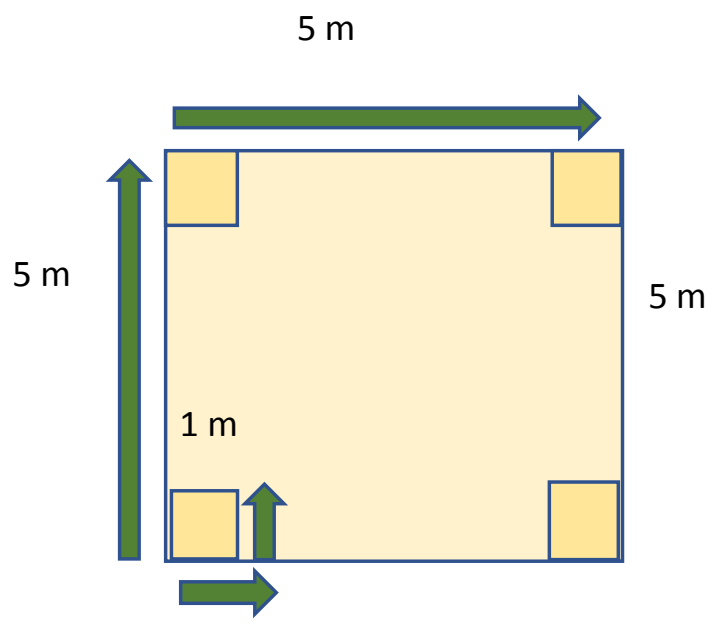

Figura 1 .- División de parcelas 
En la figura 1. Se indica cada uno de los cuadrantes de $1 \mathrm{~m} \times 1 \mathrm{~m}$, los mismos que se subdividieron en celdillas de $0.1 \mathrm{~m}$ x $0.1 \mathrm{~m}$ para lo cual se utilizó un armazón de madera y un enrejado de hilos que delimitaron un total de 100 celdillas esto permitió la toma de datos de cada subparcela para su posterior análisis.

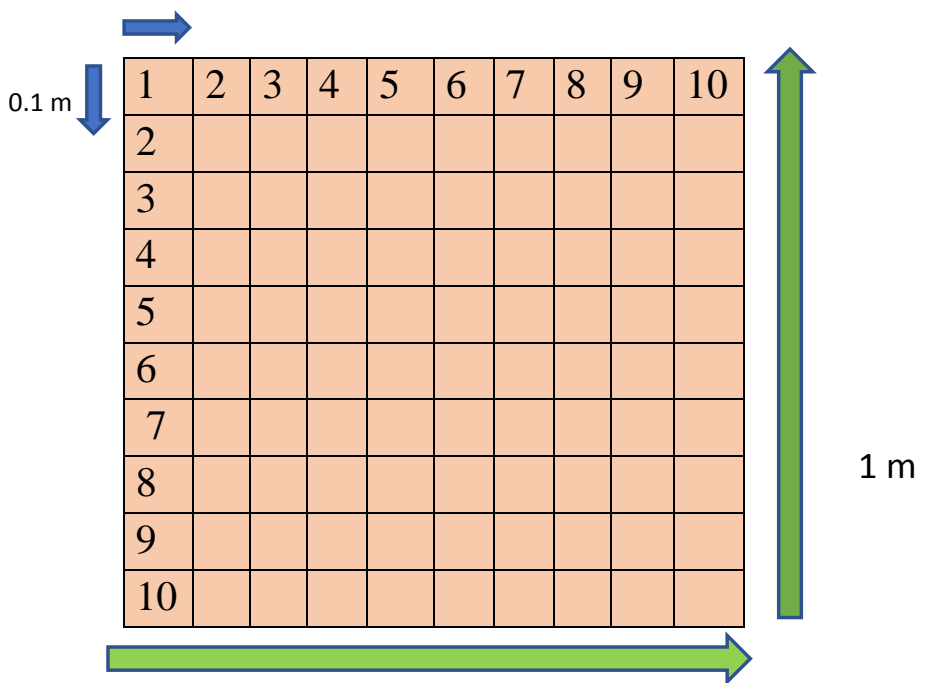

Figura 2.- Diseño de cuadrantes

\section{Extracción de especies y herborización}

Las especies vegetales que se recolectaron en la zona de estudio fueron herborizadas en el mismo lugar con papel periódico y a su vez prensadas para su transportación, las especies fueron secadas manualmente bajo sombra y prensadas, se cambió de papel periódico cada día para evitar putrefacción de las muestras o que estas se llenen de hongos.

Las muestras fueron identificadas en el herbario de la ESPOCH, a su vez el herbario emitió un certificado de reconocimiento de muestras vegetales.

\section{Tabulación de datos}

Se procedió a contar y registrar los datos en el cuaderno de campo; se registró el número de individuos, altura y cobertura de cada especie, con el fin de obtener datos cuantitativos de la vegetación. Las especies que no se pudieron identificar en el campo fueron registradas con códigos e identificadas en el herbario de la ESPOCH. Ya reconocidas las especies se procedió a realizar el listado con sus respectivos datos para los cálculos respectivos.

\section{Cálculo de datos}

Con la información obtenida se determinaron valores de importancia por especie y familia, densidad relativa, frecuencia relativa e índices de diversidad. 


\section{IVI: (Índice de valor de importancia)}

$\mathrm{IVI}=\mathrm{DR}+\mathrm{FR}$

DR = Densidad Relativa

$\mathrm{DR}=$ (Número de individuos de una especie / número total de individuos en el muestreo) $\mathbf{X}$ 100.

\section{FR $=$ Frecuencia Relativa}

$\mathrm{FR}=$ (Número de unidades de muestreo con la especie / Sumatoria de las frecuencias de todas las especies) $\mathbf{X} 100$

Para este estudio el número de unidades de muestreo son 20 cuadrantes de $1 \mathrm{~m}^{2}$.

$\mathbf{D}=$ área de cobertura de la especie / área muestreada

Dr $=$ (Área de cobertura de la especie / área de cobertura de todas las especies) X 100

Índice de Shannon - Weaver

$$
H=-\sum_{\mathrm{i}=1}^{\mathrm{S}}(\mathrm{Pi}) \text { (longnPi) }
$$

\section{Donde:}

$\mathrm{H}=$ Índice de Shannon

$\mathrm{S}=$ Número de especies

$\mathrm{Pi}$ = Proporción del número total de individuos que constituyen la especie (Velásquez, 1997). Índice de Simpson

$$
I S D=1-\sum(\mathrm{Pi})^{2}
$$

\section{Donde:}

ISD $=$ Índice de Simpson

$\mathrm{Pi}$ = Proporción del número total de individuos que constituyen la especie.

Los índices de Shannon y Simpson toman en consideración tanto la riqueza como la equitividad de especies.

Tabla 2. Interpretación de la diversidad:

\begin{tabular}{|l|l|}
\hline Valores & Interpretación \\
\hline $0,00-0,35$ & Diversidad baja \\
\hline $0.36-0.75$ & Diversidad mediana \\
\hline $0.76-1,00$ & Diversidad alta \\
\hline
\end{tabular}

Fuente: (Pujos, 2013)

Índice de Sorensen

$$
\text { Iss }=\frac{2 C}{A+B} \times 100
$$

Donde: 
Iss=Índice de Sorensen

A = Número de especies en el sitio 1

$\mathrm{B}=$ Número de especies en el sitio 2

$\mathrm{C}=$ Número de especies similares presentes en ambos sitios A y B.

Tabla 3. Interpretación de Similitud

\begin{tabular}{|c|c|}
\hline Valores & Interpretación \\
\hline $\mathbf{0 , 0 0 - 0 , 3 5}$ & Disimiles \\
\hline $\mathbf{0 , 3 6 - 0 , 7 0}$ & Medianamente similares \\
\hline $\mathbf{0 . 7 1 - \mathbf { 1 0 0 }}$ & Muy similares \\
\hline
\end{tabular}

Fuente: (Ordoñez, 1999).

Resultados.

Especies registradas en el área de estudio

Tabla 4. Vegetación Registrada en el Páramo Machay

\begin{tabular}{|l|l|l|l|l|}
\hline $\mathbf{N}^{\circ}$ & FAMILIA & NOMBRE CIENTÍFICO & Ge & Sp \\
\hline $\mathbf{1}$ & Apiaceae & $\begin{array}{l}\text { Azorella pedunculata (Spreng.) Mathias \& } \\
\text { Constance. }\end{array}$ & & \\
\hline $\mathbf{2}$ & Apiaceae & Daucus montanus Humb et Bonpl. ex spreng & 3 & 3 \\
\hline $\mathbf{3}$ & Apiaceae & Eryngium humile Cav & & \\
\hline $\mathbf{4}$ & Rosaceae & Lachemilla orbiculata (Ruiz \& Pav.) Rydb. & 1 & 1 \\
\hline $\mathbf{5}$ & Fabaceae & Lupinus sp. & 2 & 2 \\
\hline $\mathbf{6}$ & Fabaceae & Trifolium repens L. & & \\
\hline $\mathbf{7}$ & Poaceae & Festuca cf & & \\
\hline $\mathbf{8}$ & Poaceae & Calamagrostis intermedia (J. Presl) Steud. & & \\
\hline $\mathbf{9}$ & Poaceae & Bromus pitensis Kunth & & \\
\hline $\mathbf{1 0}$ & Poaceae & Holcus lanatus L. & 5 & 6 \\
\hline $\mathbf{1 1}$ & Poaceae & Dactylis glomerata L. & & \\
\hline $\mathbf{1 2}$ & Poaceae & Festuca sp. & 1 & 1 \\
\hline $\mathbf{1 3}$ & Cyperaceae & Carex bonplandii Kunth & 1 & 2 \\
\hline $\mathbf{1 4}$ & Plantaginaceae & Plantago linearis var. & & \\
\hline $\mathbf{1 5}$ & Plantaginaceae & Plantago serícea Ruíz \& Pav. & & \\
\hline $\mathbf{1 6}$ & Asteraceae & Hypochaeris sessiliflora Kunth & & \\
\hline $\mathbf{1 7}$ & Asteraceae & Baccharis caespitosa (Lam.) Pers & & \\
\hline $\mathbf{1 8}$ & Asteraceae & Gnaphalium elegans Kunth. & & \\
\hline $\mathbf{1 9}$ & Asteraceae & Taraxacum officinale Weber & Werneria nubigena Kunth & \\
\hline $\mathbf{2 0}$ & Asteraceae & & & \\
\hline
\end{tabular}




\begin{tabular}{|l|l|l|l|l|}
\hline $\mathbf{2 1}$ & Asteraceae & Lasiocephalus ovatus Schltdl. & 11 & 11 \\
\hline $\mathbf{2 2}$ & Asteraceae & Gynoxys buxifolia (Kunth) Cass & & \\
\hline $\mathbf{2 3}$ & Asteraceae & Bidens andicola Kunth & & \\
\hline $\mathbf{2 4}$ & Asteraceae & Casiocaptalus (Schltdl.) B. Nord & & \\
\hline $\mathbf{2 5}$ & Asteraceae & Erigeron ecuadoriensis Hieron & & \\
\hline $\mathbf{2 6}$ & Asteraceae & Asteracea & & \\
\hline $\mathbf{2 7}$ & Melastomataceae & Brachyotum ledifolium (Desr.) Triana & 1 & 1 \\
\hline $\mathbf{2 8}$ & Ericaceae & Pernettya prostrata (Cav.) DC & & \\
\hline $\mathbf{2 9}$ & Ericaceae & Vaccinium floribundum Kunth & 3 & 3 \\
\hline $\mathbf{3 0}$ & Ericaceae & Disterigma empetrifolium (Kunth) Drude. & & \\
\hline $\mathbf{3 1}$ & Hypericaceae & Hipericum laricifolium Juss. & 1 & 1 \\
\hline $\mathbf{3 2}$ & Lycopodiaceae & Huperzia crassa (Humb. \& Bonpl. Ex Willd.) & 1 & 1 \\
\hline $\mathbf{3 3}$ & Polygonaceae & Rumex acetosella L. & 1 & 1 \\
\hline $\mathbf{3 4}$ & Geraniaceae & Geranium laxicaule R. Knuth & 1 & 2 \\
\hline $\mathbf{3 5}$ & Geraniaceae & Geranium L. & & \\
\hline $\mathbf{3 6}$ & Ranunculaceae & $\begin{array}{l}\text { Ranunculus praemorsus Humb., Bonpl. \& Kunth ex } \\
\text { DC. }\end{array}$ & 1 & 1 \\
\hline $\mathbf{3 7}$ & Gentianaceae & Gentiana sedifolia Kunth & & \\
\hline $\mathbf{3 8}$ & Gentianaceae & Gentianella cerastioides (Kunth) Fabris. & 2 & 2 \\
\hline $\mathbf{3 9}$ & Caprifoliaceae & Phyllactis rigida (Ruiz \& Pav.) Pers. & 2 & 2 \\
\hline $\mathbf{4 0}$ & Caprifoliaceae & Valeriana microphylla Kunth & 1 & 1 \\
\hline $\mathbf{4 1}$ & Liquen & Indeterminada 1 & 36 & 40 \\
\hline $\mathbf{4 2}$ & Musgo & Indeterminada & & \\
\hline & TOTAL 18 & & 1 & 1 \\
\hline
\end{tabular}

Fuente: Investigación primaria, 2018.

En el estudio realizado se registraron los datos de las especies vegetales de 20 cuadrantes de $1 \mathrm{~m}^{2}$ cada uno, estos cuadrantes están ubicados en 5 parcelas transitorias de $5 \mathrm{~m}$ X $5 \mathrm{~m}$ las cuales están distribuidas al azar en la zona de estudio. Se colectó 40 muestras de plantas vasculares terrestres, correspondientes a 16 familias botánicas, 36 géneros y 40 especies, además también se colectó 1 liquen y 1 musgo de familia, género y especie no identificado lo que suman 42 muestras vegetales las cuales han sido identificadas en el Herbario de la ESPOCH.

Tabla 5. Resumen de la composición Florística de las 5 parcelas

\begin{tabular}{|l|l|l|l|l|l|}
\hline Parcelas & P. $\mathbf{N}^{\circ} \mathbf{- 0 1}$ & P. $\mathbf{N}^{\circ} \mathbf{- 0 2}$ & P. $\mathbf{N}^{\circ} \mathbf{- 0 3}$ & P. $\mathbf{N}^{\circ} \mathbf{- 0 4}$ & P. $\mathbf{N}^{\circ} \mathbf{- 0 5}$ \\
\hline Familias & 15 & 14 & 16 & 14 & 14 \\
\hline Géneros & 24 & 23 & 30 & 25 & 27 \\
\hline Especies & 27 & 23 & 30 & 25 & 29 \\
\hline
\end{tabular}

Fuente: Investigación primaria, 2018. 
En la tabla 5, se aprecia mayor riqueza en la parcela 03. Con 16 familias, 30 géneros y 30 especies. Se acota a este resultado lo que el autor Pujos, (2013) menciona que los sitios menos perturbados son más ricos en especies, en los resultados obtenidos la parcela 03 presenta 16 familias y 30 especies lo que según los resultados es la parcela con más familias y especies. Por otro lado, la parcela 02 presenta 14 familias y 23 especies siendo la parcela que menos diversidad tiene lo cual se explica por la presencia de ganado vacuno, incendios y otras perturbaciones provocadas por el hombre. No obstante, la parcela 05 también presenta 14 familias, pero el número de especie es mayor que en las parcelas 01, 02, 04, lo cual cumple con la lógica de menor perturbación mayor diversidad mencionada anteriormente. Cabe reconocer que esta zona de estudio se encuentra en proceso de recuperación por las actividades antrópicas del hombre mencionadas anteriormente.

\section{Descripción del Índice de Valor de Importancia (I.V.I) de especies de las cinco parcelas} Las especies con mayor valor de importancia en este caso es el Musgo (Indeterminada) con un 30\% esta especie se encuentra en forma de un tipo de colchón en asociación con la mayoría de especies y sola formando grandes cubiertas esponjosas sobre el suelo de páramo, la especie Brachyotum ledifolium (Desr.) Triana, y Gynoxys buxifolia (Kunth) Cass con 5 \% 4,25 \% es la segunda especie dominante en este ecosistema lo cual concuerda con el estudio realizado por (Caluña V, 2017) quien concuerdan con lo dicho por Freire F, 2004 en tercer lugar tenemos a Calamagrostis intermedia (J.Presl) Steud con el 3.18\% esta especie es propia de los páramos de pajonal según la clasificación de Proyecto Páramo (1999). Por otra parte, la especie Lasiocephalus ovatus Schltdl, es propia de los páramos sin embargo en este sitio de estudio los individuos son muy pocos, a la igual que otras especies también registradas en este ecosistema.

\section{Índice Shannon-Weaver}

Tabla 6. Descripción del índice de Shannon-Weaver

\begin{tabular}{|l|l|l|l|}
\hline $\mathbf{N}^{\circ}$ - de Parcela & $\begin{array}{l}\text { Valor } \\
\text { Calculado }\end{array}$ & Valor Referencial & Interpretación \\
\hline 01 & 0.613 & $0.36-0.75$ & Diversidad Media \\
\hline 02 & 0.127 & $0.0-0.35$ & Diversidad Baja \\
\hline 03 & 0.652 & $0.36-0.75$ & Diversidad Media \\
\hline 04 & 0.229 & $0.0-0.35$ & Diversidad Baja \\
\hline 05 & 1.0 & $0.76-1.00$ & Diversidad Alta \\
\hline
\end{tabular}

Fuente: Investigación secundaria, 2018.

\section{Descripción de resultados Índice de Diversidad de Shannon-Weaver}

Los resultados obtenidos del Índice de diversidad de Shannon-Weaver en las 5 parcelas demuestran que la parcela 02 y 04 tienen una diversidad baja de acuerdo a la tabla de 
interpretación mencionada por (Smith \& Smith, 2007) el cual tiene un rango de $0.0-0.35$ el cual concuerda con los resultados obtenidos. Mientras que las parcelas 01 y 03 tienen una diversidad media de acuerdo a la tabla de interpretación mencionada por (Smith \& Smith, 2007) el cual tiene un rango de $0.36-0.75$ valores que se encuentran como extremos de los resultados obtenidos luego de aplicar la fórmula mencionada por (Londo,J. 2018). La parcela 05 fue la parcela que mayor índice de diversidad tuvo pues según los resultados obtenidos y comparados con la tabla de interpretación mencionada por (Smith \& Smith, 2007) es una parcela que posee una diversidad alta dentro de esta zona de estudio.

\section{Índice de Simpson}

Tabla 7. Descripción del Î́ndice de Simpson

\begin{tabular}{|l|l|l|l|}
\hline $\mathrm{N}^{\circ}$ - de Parcela & Valor Calculado & Valor Referencial & Interpretación \\
\hline 1 & 0.25 & $0.0-0.35$ & Diversidad Baja \\
\hline 2 & 0.034 & $0.0-0.35$ & Diversidad Baja \\
\hline 3 & 0.222 & $0.0-0.35$ & Diversidad Baja \\
\hline 4 & 0.073 & $0.0-0.35$ & Diversidad Baja \\
\hline 5 & 0.456 & $0.36-0.75$ & Diversidad Media \\
\hline
\end{tabular}

Fuente: Investigación secundaria, 2018.

\section{Descripción de Resultados del Índice de diversidad de Simpson}

Los resultados obtenidos del Índice de Simpson en las 5 parcelas son similares a los obtenidos en el índice se Shannon - Weaver o que dé demuestra que este ecosistema tiene un ecosistema de una diversidad media según los resultados obtenidos tanto en Simpson como en Shannon - Weaver se acercan a 1 valor máximo de la tabla de interpretación mencionada por (Smith \& Smith, 2007) aplica para los dos índices de diversidad dieron como resultado que la parcela $\mathrm{N}^{\circ}$ - 05 es la más diversa dentro del estudio. Los resultados además se corroboran con la observación directa que se realizó en el sitio de estudio.

Similitud de acuerdo al Índice de Sorensen

Tabla 8. Resultados Índice de Similitud de Sorensen

\begin{tabular}{|l|l|l|l|l|}
\hline $\begin{array}{l}\mathbf{N}^{\circ}- \\
\text { Parcela }\end{array}$ & $\begin{array}{l}\text { Especies } \\
\text { comunes }\end{array}$ & $\begin{array}{l}\text { Valor } \\
\text { Calculado }\end{array}$ & $\begin{array}{l}\text { Valor } \\
\text { Referencial }\end{array}$ & Interpretación \\
\hline P1 vs P2 & 17 & $68 \%$ & $0.36-0.70$ & $\begin{array}{l}\text { Medianamente } \\
\text { similares }\end{array}$ \\
\hline P1 vs P3 & 19 & $66.67 \%$ & $0.36-0.70$ & $\begin{array}{l}\text { Medianamente } \\
\text { similares }\end{array}$ \\
\hline P1 vs P4 & 13 & $50 \%$ & $0.36-0.70$ & $\begin{array}{l}\text { Medianamente } \\
\text { similares }\end{array}$ \\
\hline P1 vs P5 & 22 & $78.57 \%$ & $0.71-1.00$ & Muy similares \\
\hline
\end{tabular}




\begin{tabular}{|l|l|l|l|l|}
\hline P2 vs P3 & 17 & $64.15 \%$ & $0.36-0.70$ & $\begin{array}{l}\text { Medianamente } \\
\text { similares }\end{array}$ \\
\hline P2 vs P4 & 15 & $62.5 \%$ & $0.36-0.70$ & $\begin{array}{l}\text { Medianamente } \\
\text { similares }\end{array}$ \\
\hline P2 vs P5 & 17 & $65.38 \%$ & $0.36-0.70$ & $\begin{array}{l}\text { Medianamente } \\
\text { similares }\end{array}$ \\
\hline P3 vs P4 & 17 & $61.82 \%$ & $0.36-0.70$ & $\begin{array}{l}\text { Medianamente } \\
\text { similares }\end{array}$ \\
\hline P3 vs P5 & 23 & $77.97 \%$ & $0.71-1.00$ & Muy similares \\
\hline P4 vs P5 & 14 & $51.85 \%$ & $0.36-0.70$ & $\begin{array}{l}\text { Medianamente } \\
\text { similares }\end{array}$ \\
\hline
\end{tabular}

Fuente: Investigación secundaria, 2018.

\section{Descripción de resultados del Índice de Similitud de Sorensen}

El índice de similitud de Sorensen ayuda a determinar la presencia o ausencia de las especies (Smith \& Smith. 2001). Al comparar los valores de similitud entre parcelas se observa que la vegetación es medianamente similar en más del 50 \% lo que quiere decir que la mayoría de las especies se pueden encontrar en toda la zona de estudio. La comparación que se realizó entre las parcelas P3 vs P5 y P1 vs P5 dieron como resultado que las parcelas son muy similares de acuerdo la tabla de comparación propuesta por (Mostacedo, 2000).

Presencia de especies de las 5 parcelas

Tabla 9. Descripción de la presencia de especies entre las 5 parcelas

\begin{tabular}{|l|l|l|l|l|l|l|l|}
\hline $\mathbf{N}^{\circ}$ & FAMILIA & NOMBRE CIENTíFICO & $\mathbf{1}$ & $\mathbf{2}$ & $\mathbf{3}$ & $\mathbf{4}$ & $\mathbf{5}$ \\
\hline $\mathbf{1}$ & Apiaceae & $\begin{array}{l}\text { Azorella pedunculata (Spreng.) Mathias \& } \\
\text { Constance. }\end{array}$ & $\mathrm{X}$ & $\mathrm{X}$ & $\mathrm{X}$ & $\mathrm{X}$ \\
\hline $\mathbf{2}$ & Apiaceae & Daucus montanus Humb et Bonpl. ex spreng & $\mathrm{X}$ & & & $\mathrm{X}$ & \\
\hline $\mathbf{3}$ & Apiaceae & Eryngium humile Cav & $\mathrm{X}$ & $\mathrm{X}$ & $\mathrm{X}$ & & $\mathrm{X}$ \\
\hline $\mathbf{4}$ & Rosaceae & Lachemilla orbiculata (Ruiz \& Pav.) Rydb. & $\mathrm{X}$ & $\mathrm{X}$ & $\mathrm{X}$ & $\mathrm{X}$ & $\mathrm{X}$ \\
\hline $\mathbf{5}$ & Fabaceae & Lupinus sp. & $\mathrm{X}$ & $\mathrm{X}$ & $\mathrm{X}$ & & $\mathrm{X}$ \\
\hline $\mathbf{6}$ & Fabaceae & Trifolium repens L. & & & $\mathrm{X}$ & $\mathrm{X}$ & \\
\hline $\mathbf{7}$ & Poaceae & Festuca cf & $\mathrm{X}$ & $\mathrm{X}$ & $\mathrm{X}$ & & $\mathrm{X}$ \\
\hline $\mathbf{8}$ & Poaceae & Calamagrostis intermedia (J. Presl) Steud. & $\mathrm{X}$ & $\mathrm{X}$ & $\mathrm{X}$ & $\mathrm{X}$ & $\mathrm{X}$ \\
\hline $\mathbf{9}$ & Poaceae & Bromus pitensis Kunth & $\mathrm{X}$ & $\mathrm{X}$ & $\mathrm{X}$ & & $\mathrm{X}$ \\
\hline $\mathbf{1 0}$ & Poaceae & Holcus lanatus L. & $\mathrm{X}$ & & $\mathrm{X}$ & $\mathrm{X}$ & $\mathrm{X}$ \\
\hline $\mathbf{1 1}$ & Poaceae & Dactylis glomerata L. & & $\mathrm{X}$ & & \\
\hline $\mathbf{1 2}$ & Poaceae & Festuca sp. & $\mathrm{X}$ & & & & \\
\hline $\mathbf{1 3}$ & Cyperaceae & Carex bonplandii Kunth & $\mathrm{X}$ & & $\mathrm{X}$ & & $\mathrm{X}$ \\
\hline
\end{tabular}


ISSN: 2602-8085

www.cienciadigital.org

Vol. 3, N³.1 p. 326-340, julio - septiembre, 2019

\begin{tabular}{|c|c|c|c|c|c|c|c|}
\hline 14 & Plantaginaceae & Plantago linearis var. & $x$ & & $x$ & & $\mathrm{x}$ \\
\hline 15 & Plantaginaceae & Plantago serícea Ruíz \& Pav. & $x$ & & & & $x$ \\
\hline 16 & Asteraceae & Hypochaeris sessiliflora Kunth & $x$ & $x$ & $x$ & $x$ & $\mathrm{x}$ \\
\hline 17 & Asteraceae & Baccharis caespitosa (Lam.) Pers & $x$ & $x$ & $x$ & $x$ & $\mathrm{x}$ \\
\hline 18 & Asteraceae & Gnaphalium elegans Kunth. & $x$ & & & & $\mathrm{x}$ \\
\hline 19 & Asteraceae & Taraxacum officinale Weber & & $x$ & $x$ & & $\mathrm{x}$ \\
\hline 20 & Asteraceae & Werneria nubigena Kunth & & & $x$ & $x$ & $\mathrm{x}$ \\
\hline 21 & Asteraceae & Lasiocephalus ovatus Schltdl. & & & & $x$ & \\
\hline 22 & Asteraceae & Gynoxys buxifolia (Kunth) Cass & & & $x$ & $x$ & \\
\hline 23 & Asteraceae & Bidens andicola Kunth & & & $x$ & $x$ & $\mathrm{x}$ \\
\hline 24 & Asteraceae & Casiocaptalus (Schltdl.) B. Nord & & $x$ & & $\mathrm{X}$ & $\mathrm{x}$ \\
\hline 25 & Asteraceae & Erigeron ecuadoriensis Hieron & & & $\mathrm{X}$ & & $\mathrm{x}$ \\
\hline 26 & Asteraceae & Asteracea & & $x$ & $X$ & $\mathrm{X}$ & $\mathrm{X}$ \\
\hline 27 & Melastomataceae & Brachyotum ledifolium (Desr.) Triana & & $x$ & $X$ & $\mathrm{x}$ & \\
\hline 28 & Ericaceae & Pernettya prostrata (Cav.) DC & $x$ & $x$ & $x$ & $\mathrm{x}$ & $x$ \\
\hline 29 & Ericaceae & Vaccinium floribundum Kunth & $x$ & $x$ & & $\mathrm{X}$ & \\
\hline 30 & Ericaceae & Disterigma empetrifolium (Kunth) Drude. & & $x$ & & $\mathrm{X}$ & \\
\hline 31 & Hypericaceae & Hipericum laricifolium Juss. & $x$ & $x$ & & $\mathrm{X}$ & \\
\hline 32 & Lycopodiaceae & Huperzia crassa (Humb. \& Bonpl. Ex Willd.) & $x$ & $x$ & & $\mathrm{X}$ & $\mathrm{x}$ \\
\hline 33 & Polygonaceae & Rumex acetosella $L$. & $\mathrm{X}$ & & $\mathrm{X}$ & & $\mathrm{x}$ \\
\hline 34 & Geraniaceae & Geranium laxicaule R. Knuth & $x$ & $x$ & $x$ & & $\mathrm{x}$ \\
\hline 35 & Geraniaceae & Geranium L. & $x$ & & & $\mathrm{X}$ & $\mathrm{x}$ \\
\hline 36 & Ranunculaceae & $\begin{array}{l}\text { Ranunculus praemorsus Humb., Bonpl. \& } \\
\text { Kunth ex DC. }\end{array}$ & & $x$ & $x$ & $\mathrm{X}$ & \\
\hline 37 & Gentianaceae & Gentiana sedifolia Kunth & $x$ & $x$ & $\mathrm{X}$ & & $\mathrm{X}$ \\
\hline 38 & Gentianaceae & Gentianella cerastioides (Kunth) Fabris. & & & & & $\mathrm{x}$ \\
\hline 39 & Caprifoliaceae & Phyllactis rigida (Ruiz \& Pav.) Pers. & $x$ & $x$ & $x$ & & $\mathrm{x}$ \\
\hline 40 & Caprifoliaceae & Valeriana microphylla Kunth & $x$ & $x$ & $x$ & $x$ & \\
\hline 41 & Liquen & Indeterminada 1 & & & $x$ & $x$ & \\
\hline 42 & Musgo & Indeterminada & $x$ & $x$ & $x$ & $x$ & $\mathrm{x}$ \\
\hline
\end{tabular}

Fuente: Investigación Primaria, 2018.

\section{Conclusiones.}

- La vegetación de la zona de estudio es representativa del ecosistema páramo en el análisis realizado se muestran familias como (Apiaceae, Asteraceae, Caprifoliaceae, Cyperaceae, Ericaceae, Fabaceae, Gentianaceae, Geraniaceae, Hypericaceae, Liquen, Lycopodiaceae, Melastomataceae, Musgo, Plantaginaceae, Poaceae, Polygonaceae, Ranunculaceae, Rosaceae) y especies (Azorella pedunculata (Spreng.) Mathias \& 
Constance, Baccharis caespitosa (Lam.) Pers, Calamagrostis intermedia (J.Presl) Steud, Daucus montanus Humb et Bonpl. ex spreng, Disterigma empetrifolium (Kunth) Drude, Erigeron ecuadoriensis Hieron, Eryngium humile Cav, Gentiana sedifolia Kunth, Gentianella cerastioides (Kunth) Fabris, Gnaphalium elegans Kunth, Gynoxys buxifolia (Kunth) Cass, Lachemilla orbiculata (Ruiz \& Pav.) Rydb, Lasiocephalus ovatus Schltdl, Plantago serícea Ruíz \& Pav, Werneria nubigena Kunth.entre otras), típicas de estos ecosistemas a pesar de que este páramo ha tenido gran influencia antrópica en años anteriores y el ecosistema se encuentre en proceso de recuperación.

- El Musgo (Indeterminada) se encontró en todas las parcelas y cuadrantes teniendo mayores individuos en la Parcela 2, esta especie forma una autentica alfombra viva que cubre el suelo del páramo, la especie se encuentra en perfecta asociación con los demás individuos y no impide el desarrollo de las demás especies a su alrededor.

- La composición florística no presenta una tendencia pronunciada de disminución o incremento en su vegetación, pese a que las parcelas no se encuentran en a la misma altura lo que hace pensar que la altura no tiene gran influencia en esta zona de estudio.

- La mayoría de especies se encuentran en todas las parcelas, aunque en diferente número de individuos, es decir la vegetación es casi homogénea en este páramo.

\section{Referencias bibliográficas.}

Alvarado, E., \& Gavilanes, A. (2012). Línea base de los cinco humedales de la comunidad Chocaví- páramo del Igualata. (Tesis de grado. de Ingeniero Forestal). Escuela Superior Politécnica de Chimborazo. Riobamba. .

Baquero, E., Sierra, R., Ordóñez, M., \& Tipán, L. (2004). La Vegetación de los Andes del Ecuador. Quito: Memoria explicativa de los mapas de vegetación: potencial y remanente.

Bernal, F., Sánchez, \& Zapatta. (2000). Manejo de Páramos y Zonas de Altura. QuitoEcuador: CAMAREN-IEDECA. Quito.

Londo, J. (2017). Inventario y Morfología de las Especies Forestales del Bosque Nativo Sachafilo, Ubicado en la Parroquia San Antonio de Pasa, Cantón Ambato, Provincia de Chimborazo. Tesis de grado. Escuela Superior Politécnica de Chimborazo- Riobamba.

Medina, \& Mena . (2001). Los páramos en el Ecuador.

Ministerio del Ambiente del Ecuador.[MAE]. (2013). Sistema de clasificación de los ecosistemas del Ecuador continental. (S. d. Natural., Ed.) Quito- Ecuador .

Ordoñez, J. (1999). Captura de carbono en un bosque templado: el caso de San Juan Nuevo, Michoacán. México DF. México: SEMARNAP. 
Pauli, H., Gottfried, M., Lamprecht, A., Niessner, Rumpf, S., Winkler, M., . . . Grabherr,. (2015). Manual para el trabajo de campo del proyecto GLORIA. Aproximación al estudio de las cimas. Métodos básicos, complementarios y adicionales. $5^{\text {a }}$ edición. GLORIA - Coordinación, Academia Austriaca de Ciencias y Universidad de Recursos Naturales y Ciencias de la Vida, Viena, Austria. Edición en español a cargo de Benito, J.L. \& Villar, L., Jaca,. Obtenido de https://www.researchgate.net/profile/Jose_Luis_Benito_Alonso/publication/282567 915_Manual_para_el_trabajo_de_campo_del_proyecto_GLORIA_Aproximacion_a 1_estudio_de_las_cimas_Metodos_basico_complementarios_y_adicionales_5_edici on/links/5615380308ae4ce3cc6526

Plan de Desarrollo y Ordenamiento Territorial de la Parroquia de ILAPO (PD Y OT). (2016). Gobierno Autónomo Desentralizado de la parroquia Rural Ilapo . Guano -Ecuador .

Pujos. (2013). Métodos para el análisis de datos: una aplicación para resultados provenientes de caracterizaciones de biodiversidad. .

Velásquez, A. (1997). Métodos para el análisis de datos: una aplicación para resultados provenientes de caracterizaciones de biodiversidad. . Recuperado el 12 de 02 de 2019, de http://www.bio-nica.info/biblioteca/humboldtanalisisdatos.pdf

\section{【L Ciencia}


PARA CITAR EL ARTÍCULO INDEXADO.

Paguay Tingo, M., Román Robalino, D., \& Cevallos Rodríguez, J. (2019). Composición y diversidad de especies forestales en el páramo Machay- ChimborazoEcuador. Ciencia Digital, 3(3.1), 326-340.

https://doi.org/10.33262/cienciadigital.v3i3.1.706

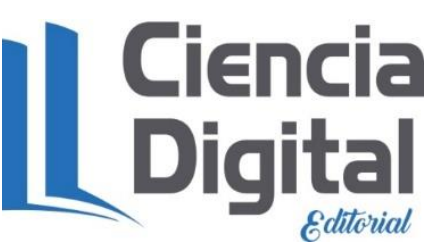

El artículo que se publica es de exclusiva responsabilidad de los autores y no necesariamente reflejan el pensamiento de la Revista Explorador Digital.

El articulo queda en propiedad de la revista y, por tanto, su publicación parcial y/o total en otro medio tiene que ser autorizado por el director o editor de la Revista Explorador Digital. 